\title{
THE MOLONGLO 843 MHz SURVEY OF THE MAGELLANIC CLOUDS
}

\author{
A.J. TURTLE, S.W. AMY \\ School of Physics \\ University of Sydney NSW 2006 \\ Australia
}

\begin{abstract}
A radio survey of the Magellanic Clouds (MCs) at $843 \mathrm{MHz}$ has been completed and is being prepared for publication. The survey was made with the Molonglo Observatory Synthesis Telescope (MOST) operated by the Astrophysics Department, University of Sydney. This survey of the Clouds has a high angular resolution ( $45 \mathrm{arcsec}$ ) and a sensitivity of a few mJy/beam even for objects of large angular extent
\end{abstract}

\section{Introduction}

The MOST is an earth-rotation aperture synthesis instrument operating at the frequency of 843 $\mathrm{MHz}$ and receiving right-circular polarization. A fuller description is given in Mills (1981). After a single 12-hour observation a provisional map is produced of a field of $70^{\prime} \times 70^{\prime} \operatorname{cosec}|\delta|$ with a beamwidth of about 45 arcsec at the declination of the Clouds. These maps are perfectly adequate for inspection, but the data are currently being reprocessed using correction factors which will produce higher quality maps for publication. A sensitivity of a few mJy/beam has been achieved over most of the region and selected parts of the survey have been used to compile a preliminary catalogue of radio supernova remnants in the Magellanic Clouds (Mills et al. 1984). Ye (1988) has also used the survey to study HII regions in the SMC.

\section{The survey}

The regions of the SMC and LMC already observed are shown in Table 1. These extend well beyond the areas containing catalogued HII regions (Davies et al. 1976).

Table 1. The regions of the Magellanic Clouds surveyed with the MOST

\begin{tabular}{lcc}
\hline & RA range (B1950.0) & Dec range (B1950.0) \\
\hline SMC & $00^{\mathrm{h}} 25^{\mathrm{m}} \rightarrow 01^{\mathrm{h}} 35^{\mathrm{m}}$ & $-70^{\circ} 45^{\prime} \rightarrow-74^{\circ} 40^{\prime}$ \\
LMC & $04^{\mathrm{h}} 46^{\mathrm{m}} \rightarrow 05^{\mathrm{h}} 58^{\mathrm{m}}$ & $-65^{\circ} 10^{\prime} \rightarrow-72^{\circ} 20^{\prime}$ \\
\hline
\end{tabular}

114

R. Haynes and D. Milne (eds.), The Magellanic Clouds, 114-115.

(c) 1991 IAU. Printed in the Netherlands. 
Fig. 1 shows part of a standard LMC survey field after reprocessing of data but without CLEANing.

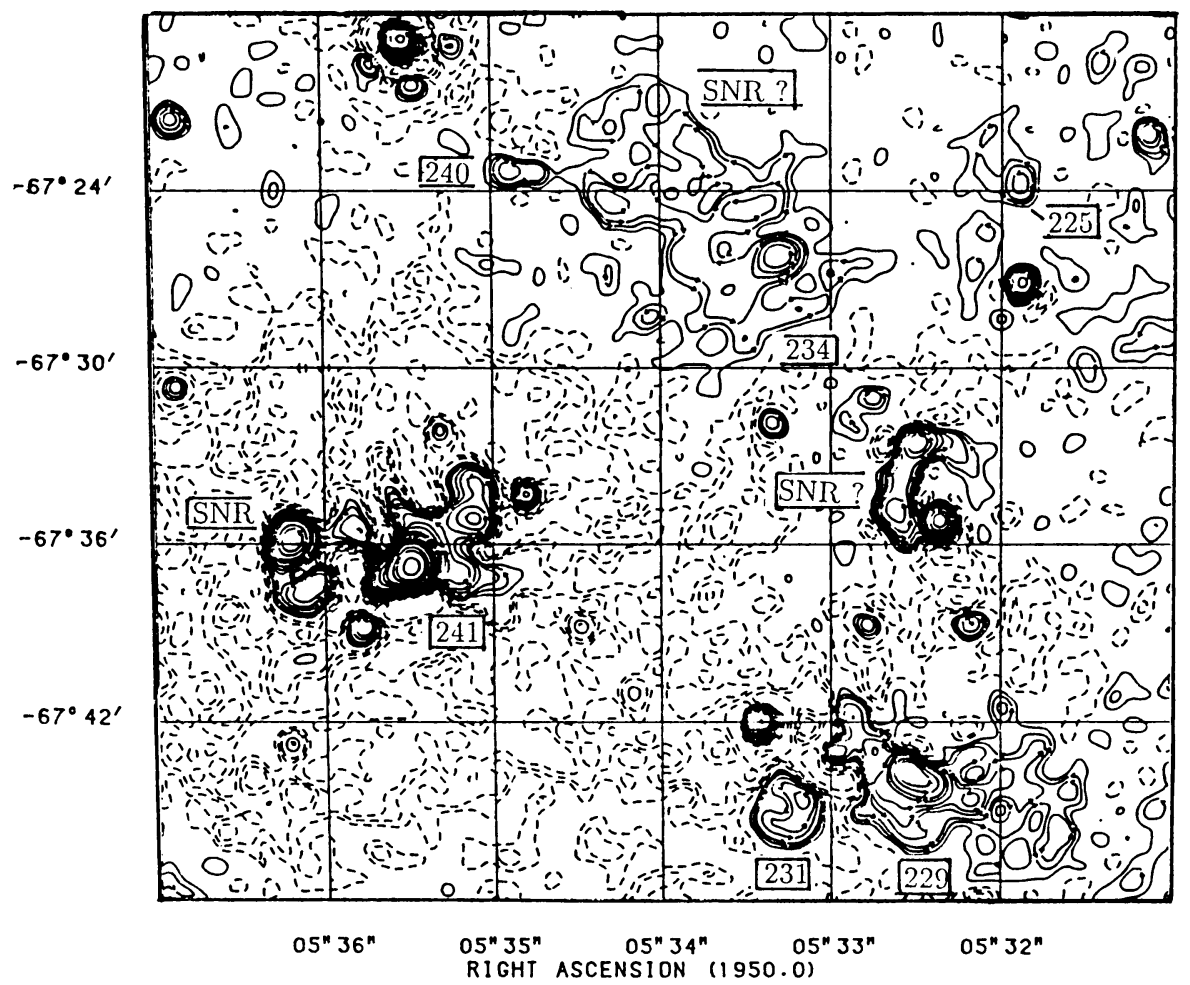

Figure 1. Part of an LMC survey field after reprocessing. The contour levels are $-10,-5,-3,-2,-1,1$, $2,3,5,7,10,15,20,30,40,60,80,100,150,200,300 \mathrm{mJy} / \mathrm{beam}$. The labelled regions correspond to those in Davies et al. (1976). The unresolved sources are probably background objects.

\section{References}

Davies, R.D., Elliott, K.H., Meaburn, J. (1976), Mem. R. astr. Soc. 81, 89.

Mills, B.Y. (1981), Proc. Astron. Soc. Aust. 4, 156.

Mills, B.Y., Turtle, A.J., Little, A.G., Durdin, J.M. (1984), Aust. J. Phys. 37, 321.

Ye, T. (1988), PhD Thesis, University of Sydney. 